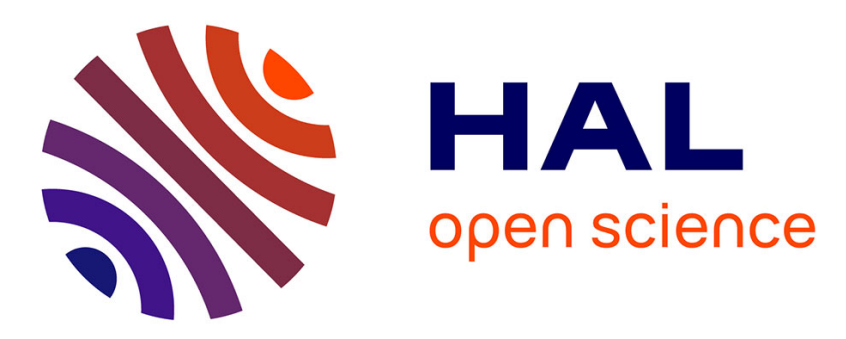

\title{
Molecular and immunological biomarkers to predict IVIg response
}

Caroline Galeotti, Srini V Kaveri, Jagadeesh Bayry

\section{To cite this version:}

Caroline Galeotti, Srini V Kaveri, Jagadeesh Bayry. Molecular and immunological biomarkers to predict IVIg response. Trends in Molecular Medicine, 2015, 21 (3), pp.145-147. 10.1016/j.molmed.2015.01.005 . hal-01117084

\section{HAL Id: hal-01117084 https://hal.sorbonne-universite.fr/hal-01117084}

Submitted on 16 Feb 2015

HAL is a multi-disciplinary open access archive for the deposit and dissemination of scientific research documents, whether they are published or not. The documents may come from teaching and research institutions in France or abroad, or from public or private research centers.
L'archive ouverte pluridisciplinaire HAL, est destinée au dépôt et à la diffusion de documents scientifiques de niveau recherche, publiés ou non, émanant des établissements d'enseignement et de recherche français ou étrangers, des laboratoires publics ou privés. 


\section{Molecular and immunological biomarkers to predict IVIg response}

\section{Caroline Galeotti ${ }^{1,2,3,4}$, Srini V Kaveri ${ }^{1,2,3,5,6}$, Jagadeesh Bayry ${ }^{1,2,3,5,6}$}

${ }^{1}$ Institut National de la Santé et de la Recherche Médicale Unité 1138, Paris, F-75006, France

${ }^{2}$ Centre de Recherche des Cordeliers, Equipe - Immunopathology and therapeutic immunointervention, Paris, F-75006, France

${ }^{3}$ Sorbonne Universités, UPMC Univ Paris 06, UMR S 1138, Paris, F-75006, France

${ }^{4}$ Department of Pediatric Rheumatology, National Referral Centre of Auto-

inflammatory Diseases, CHU de Bicêtre, le Kremlin Bicêtre, F-94270, France

${ }^{5}$ Université Paris Descartes, Sorbonne Paris Cité, UMR S 1138, Paris, F-75006, France

${ }^{6}$ International Associated Laboratory IMPACT (Institut National de la Santé et de la Recherche Médicale, France - Indian Council of Medical Research, India), National Institute of Immunohematology, Mumbai, 400012, India

Corresponding author: Jagadeesh Bayry (jagadeesh.bayry@crc.jussieu.fr) 


\begin{abstract}
Some patients with autoimmune and inflammatory diseases treated with intravenous immunoglobulin G (IVIg) as a first line therapy are refractory. Identification of predictive biomarker(s) to segregate responders and non-responders to IVIg therapy remains critical. A number of biomarkers, particularly in Kawasaki disease, have shown potential for predicting response to IVIg.
\end{abstract}

\title{
Keywords
}

intravenous immunoglobulin; cytokines; Fc $\gamma$ receptor; Kawasaki disease; GuillainBarré syndrome; immune thrombocytopenia 
Intravenous immunoglobulin (IVIg) is a pooled preparation of normal human immunoglobulin $\mathrm{G}$ (IgG) purified from the plasma of several thousand healthy donors. IVIg is one of the most highly solicited therapeutic molecules for the therapy of autoimmune and inflammatory diseases. Randomized clinical trials have demonstrated the therapeutic utility of IVIg in a wide range of diseases including Kawasaki disease (KD), immune thrombocytopenia (ITP), chronic inflammatory demyelinating polyneuropathy, anti-neutrophil cytoplasmic antibody-associatedvasculitis, myasthenia gravis, dermatomyositis, Guillain-Barré syndrome (GBS), graft-versus-host disease and others. Several mutually nonexclusive mechanisms have been proposed for IVIg that include inhibition of innate and adaptive immune cell activation and their inflammatory mediators, and induction of anti-inflammatory cells and molecules.

Nevertheless, IVIg is not a magic bullet. Even among the diseases where IVIg is recommended as a first line therapy, there are patients who do not respond. Identification of predictive biomarker(s) to segregate responders and non-responders remains a critical issue and the subject of intense research. The majority of studies have focused on KD, although other pathologies such as ITP and GBS have also been investigated.

Identifying biomarkers to predict responders to IVIg therapy is important both for patients and the health-care system. Early identification of IVIg-resistant patients helps clinicians to promptly initiate alternative therapies, reducing the morbidity and also the cost associated with IVIg therapy. Further, it would also help in preventing the empirical use of IVIg, which is currently used for nearly 100 different pathologies. 
$\mathrm{KD}$ is characterized by systemic vasculitis (mainly involving coronary arteries) in children. Preventing serious cardiac complications is the primary goal of treatment. High-dose IVIg treatment during the acute stage of KD is effective in preventing coronary artery lesions. Unfortunately, 10-20\% of patients experience IVIg resistance, which is associated with poor coronary artery outcomes. Risk-scoring systems have been developed to predict resistance to initial IVIg treatment by taking into consideration age, illness duration and various blood and biochemical parameters such as platelet and neutrophil counts, alanine aminotransferase, and C-reactive protein (CRP). Although these scores have good specificity, their low sensitivity to predict IVIg resistance in other cohorts casts doubt on their universal applicability [1].

Inflammatory mediators such as cytokines and chemokines are key players in the pathogenesis of autoimmune and inflammatory diseases. They stimulate immune and non-immune cells, program $\mathrm{T}$ cell differentiation and instruct homing of immune cells. Pre-IVIg levels of inflammatory mediators such as granulocyte-colony stimulating factor (G-CSF), IL-1 $\beta$ and IL-6 determine the responsiveness to IVIg $[2,3,4]$. The circulating levels of G-CSF were significantly higher (three-fold) in IVIg-nonresponsive patients [2] and were corroborated with significantly higher levels of polycythemia rubra vera 1 (PRV-1) and matrix metalloproteinase-8 (MMP8) $[2,3]$. As G-CSF stimulates granulopoiesis and granulocyte differentiation, these reports substantiated previous observations of significantly elevated neutrophils in IVIg-nonresponsive patients. In addition, the elevated levels of damage-associated molecular pattern molecules (DAMPs) such as high-mobility group protein B1 (HMGB1), S100A8 and S100A9 were reported to predict poor response to IVIg [3]. 
These DAMPs are released from stressed cells, and signal via receptor for advanced glycation end products (RAGE) and Toll-like receptors (TLR) to promote inflammatory responses from innate cells (Figure 1). S100A8 and A9 also regulate the adhesion of neutrophils and monocytes to endothelial cells, a process that is likely important in the vasculitis of KD.

Genetic and epigenetic studies on the pathways that signal cytokine and chemokine production have been undertaken to provide molecular insight on the IVIg response. The majority of autoimmune diseases are characterized by the presence of highaffinity autoantibodies that form immune complexes with auto-antigens and stimulate innate immune cells via $\mathrm{Fc} \gamma$ receptors $(\mathrm{Fc} \gamma \mathrm{R})$ to induce inflammatory mediators. A functional polymorphism in FCGR2A encoding an H131R substitution has been identified as a susceptibility locus for KD [5], confirming the role of Fc $\gamma \mathrm{R}$ signaling in the pathogenesis of KD. A family-based genetic study also confirmed this observation [6], but this polymorphism does not predict response to IVIg therapy.

The recent study by Kuo et al. demonstrates that it is not the polymorphism in $F C G R 2 A$, but rather epigenetic changes, in particular hypomethylation in five $\mathrm{CpG}$ sites that predicts IVIg resistance [7]. Therefore, the threshold of activation of Fc $\gamma$ RIIA determines the responsiveness to IVIg. Fc $\gamma$ RIIA is highly expressed on innate immune cells (including macrophages, dendritic cells and neutrophils), therefore stimulation via FcyRIIA leads to the secretion of various inflammatory cytokines and chemokines (Figure 1). Thus, this data provides mechanistic insight into previous reports of elevated levels of pro-inflammatory cytokines and unresponsiveness to IVIg therapy in KD. 
As the inhibitory FcyRIIB counteracts activation-associated signaling, it had been proposed that functional polymorphisms in FCGR2B might determine IVIg responders. In fact, increased $F C G R 2 B$ promoter activity due to high numbers of A alleles upstream of the translation start site of $F C G R 2 B$ was associated with IVIg response in KD patients [8]. As Fc $\gamma$ RIIA and Fc $\gamma$ RIIB are co-expressed on innate immune cells, the intensity of expression of these two receptors and their activity determine the severity of inflammatory response to $\mathrm{IgG}$ immune complexes.

Further studies are required to determine if epigenetic changes in $F C G R 2 A$ could be used to predict IVIg response in other pathologies. The signaling pathways that lead to the inflammatory response often vary among the pathologies and even patients. As IVIg is also beneficial in T-cell mediated inflammatory pathologies, Fc $\gamma$ R-related biomarkers might only prove useful in diseases where autoantibodies are implicated in the pathogenesis. High expression levels of interferon- $\gamma($ IFN- $\gamma)$ were associated with poor response to IVIg in childhood ITP [9]. Prominent peripheral mobilization of HLA-DR ${ }^{\text {high }} \mathrm{CD} 138^{\text {low }} \mathrm{CXCR} 4{ }^{\text {low }}$ immature plasma cells by day 7 post-IVIg therapy was correlated with strong clinical improvement in patients with GBS [10]. Importantly, such plasmacytosis was also observed in patients with chronic inflammatory demyelinating polyneuropathy, myasthenia gravis and inflammatory myopathies [10]. Hence, the quest for universal biomarker(s) that predict IVIg responsiveness should continue, and the search should focus on innate/adaptive immune cells and their cytokines and molecular regulators. Pre-IVIg levels of IL-17A in the circulation could be used to predict response to IVIg. Although there are differences in IVIg products from different suppliers, in general they are quite similar in therapeutic efficacy and anti-inflammatory mechanisms. Therefore, potential 
biomarkers would (and should) not vary for each IVIg product that is available for the therapy.

\section{Acknowledgments}

Supported by Institut National de la Santé et de la Recherche Médicale (INSERM), Centre National de la Recherche Scientifique (CNRS), Université Pierre et Marie Curie and Université Paris Descartes. Because of space limitations, we could only key reports that do not undermine the great value of uncited studies.

Conflict of interests: The authors declare no competing financial interests. 


\section{References}

1. Sleeper, L.A., et al. (2011) Evaluation of Kawasaki disease risk-scoring systems for intravenous immunoglobulin resistance. $J$ Pediatr 158, 831-835 e833

2. Abe, J., et al. (2008) Elevated granulocyte colony-stimulating factor levels predict treatment failure in patients with Kawasaki disease. J Allergy Clin Immunol 122, 1008-1013 e1008

3. Fury, W., et al. (2010) Transcript abundance patterns in Kawasaki disease patients with intravenous immunoglobulin resistance. Hum Immunol 71, 865873

4. Wang, Y., et al. (2013) Evaluation of intravenous immunoglobulin resistance and coronary artery lesions in relation to Th1/Th2 cytokine profiles in patients with Kawasaki disease. Arthritis Rheum 65, 805-814

5. Khor, C.C., et al. (2011) Genome-wide association study identifies FCGR2A as a susceptibility locus for Kawasaki disease. Nat Genet 43, 1241-1246

6. Shrestha, S., et al. (2012) Role of activating FcgammaR gene polymorphisms in Kawasaki disease susceptibility and intravenous immunoglobulin response. Circ Cardiovasc Genet 5, 309-316

7. Kuo, H.C., et al. (2014) DNA methylation array identifies genomic hypomethylation of FCGR2A in the susceptibility of Kawasaki disease and intravenous immunoglobulin resistance. Arthritis Rheumatol Dec 2. doi: 10.1002/art.38976. [Epub ahead of print]

8. Shrestha, S., et al. (2011) Functional FCGR2B gene variants influence intravenous immunoglobulin response in patients with Kawasaki disease. $J$ Allergy Clin Immunol 128, 677-680 
9. Mouzaki, A., et al. (2002) Expression patterns of Th1 and Th2 cytokine genes in childhood idiopathic thrombocytopenic purpura (ITP) at presentation and their modulation by intravenous immunoglobulin G (IVIg) treatment: their role in prognosis. Blood 100, 1774-1779

10. Mori, I., et al. (2008) Prominent plasmacytosis following intravenous immunoglobulin correlates with clinical improvement in Guillain-Barre syndrome. PLoS One 3, e2109. doi: 10.1371/journal.pone.0002109 


\section{Figure legend}

Figure 1. Examples of molecular and immunological biomarkers reported to predict response to IVIg therapy. The majority of the data presented in the figure has been derived from studies of patients with Kawasaki disease (KD). In the figure, putative biomarkers of poor response to IVIg are depicted in red boxes. The elevated levels of DAMPs (such as HMGB1, S100A8 and S100A9) released from stressed cells, and G-CSF, released from vascular endothelial cells and smooth muscle cells, were reported to predict poor response to IVIg. G-CSF stimulates granulopoiesis and granulocyte differentiation by signaling via STAT3. Hypomethylation in five CpG sites of $F C G R 2 A$ predicts IVIg resistance. NF- $\mathrm{KB}$ signaling activated by DAMPS via TLR and RAGE, and by IgG-immune complexes via FcyRIIA, lead to activation of innate immune cells such as neutrophils, macrophages and dendritic cells. These cells then secrete high levels of inflammatory mediators such as IL-6, IL-1 $\beta$, TNF, PRV-1 and MMP-8. IL-1 $\beta$ stimulates vascular endothelial cells to produce G-CSF. Pre-IVIg levels of these inflammatory mediators were also used to predict IVIg response. Abbreviations: DAMPs, damage associated molecular pattern molecules; G-CSF, granulocyte-colony stimulating factor; G-CSF-R, granulocyte-colony stimulating factor receptor; HMGB1, high-mobility group box-1; IVIg, intravenous immunoglobulin G; MMP-8, matrix metalloproteinase-8; NF-кB, nuclear factor kappa-light-chain-enhancer of activated B cells; PRV-1, polycythemia rubra vera-1; RAGE, receptor for advanced glycation end products; STAT-3, signal transducer and activator of transcription 3; TLR, Toll-like receptor; TNF, tumor necrosis factor. 


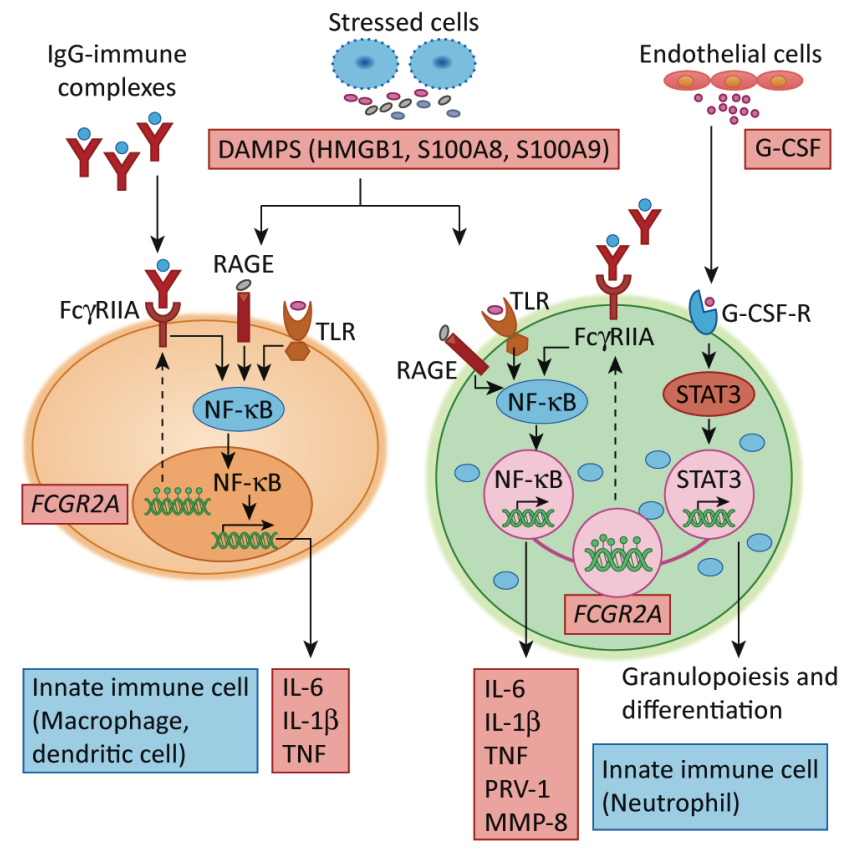

